\title{
The burden of cardiovascular morbidity in a European Mediterranean population with multimorbidity: a cross-sectional study
}

Concepción Violán 1,3* (D), Néker Bejarano-Rivera ${ }^{1,2,3,4}$, Quintí Foguet-Boreu ${ }^{1,3,5}$, Albert Roso Llorach,3, Mariona Pons-Vigués ${ }^{1,3,6}$, Miguel Martin Mateo ${ }^{3}$ and Enriqueta Pujol-Ribera ${ }^{1,3,6}$

\begin{abstract}
Background: Cardiovascular diseases are highly represented in multimorbidity patterns. Nevertheless, few studies have analysed the burden of these diseases in the population with multimorbidity. The objective of this study was to identify and describe the cardiovascular diseases among the patients with multimorbidity.

Methods: We designed a cross-sectional study in patients $\geq 19$ years old assigned to 251 primary health care centres in Catalonia, Spain. The main outcome was cardiovascular morbidity burden, defined as the presence of one or more of 24 chronic cardiovascular diseases in multimorbid patients ( $\geq 2$ chronic conditions). Two groups were defined, with and without multimorbidity; the multimorbidity group was further divided into cardiovascular and non-cardiovascular subgroups. The secondary outcomes were: modifiable major cardiovascular risk factors (smoking, hypertension, hypercholesterolaemia, diabetes) and cardiovascular risk score (REGICOR, Registre Gironi del Cor). Other variables analysed were: sex, age (19-24, 25-44, 45-64, 65-79, and 80+ years), number of chronic diseases, urban setting, active toxic habits (smoking and alcohol), physical parameters and laboratory tests.

Results: A total of 1,749,710 individuals were included (mean age, 47.4 years [SD: 17.8]; $50.7 \%$ women), of which nearly half (46.8\%) had multimorbidity (95\% Cl: 46.9-47.1). In patients with multimorbidity, the cardiovascular burden was $54.1 \%$ of morbidity ( $95 \% \mathrm{Cl}$ : 54.0-54.2) and the four most prevalent cardiovascular diseases were uncomplicated hypertension (75.3\%), varicose veins of leg (20.6\%), "other" heart disease (10.5\%) and atrial fibrillation/flutter (6.7\%). In the cardiovascular morbidity subgroup, $38.2 \%$ had more than one cardiovascular disease. The most prevalent duet and triplet combinations were uncomplicated hypertension \& lipid disorder $(38.8 \%)$ and uncomplicated hypertension \& lipid disorder \& non-insulin dependent diabetes (11.3\%), respectively. By age groups, the same duet was the most prevalent in patients aged 45-80 years and in men aged 25-44 years. In women aged 19-44, varicose veins of leg \& anxiety disorder/anxiety was the most prevalent; in men aged 19-24, it was uncomplicated hypertension \& obesity. Patients with multimorbidity showed a higher cardiovascular risk profile than the non-multimorbidity group.

Conclusions: More than $50 \%$ percent of patients with multimorbidity had cardiovascular diseases, the most frequent being hypertension. The presence of cardiovascular risk factors and the cardiovascular risk profile were higher in the multimorbidity group than the non-multimorbidity group. Hypertension, diabetes and dyslipidaemia constituted the most prevalent multimorbidity pattern.
\end{abstract}

Keywords: Multimorbidity, Cardiovascular morbidity, Cardiovascular risk factors, Comorbidity, Electronic health records, Primary health care

\footnotetext{
* Correspondence: cviolan@idiapjgol.org

'Institut Universitari d'Investigació en Atenció Primària Jordi Gol (IDIAP Jordi

Gol), Gran Via Corts Catalanes, 587 àtic, 08007 Barcelona, Spain

${ }^{3}$ Universitat Autònoma de Barcelona, Bellaterra (Cerdanyola del Vallès),

Barcelona, Spain

Full list of author information is available at the end of the article
} 


\section{Background}

Multimorbidity (MM), understood as the diagnosis of two or more disease diagnoses in the same patient, will become a major public challenge in the coming decades. A systematic review found that the prevalence of MM varied depending on age, and ranged from $13 \%$ in participants aged 18 years and older to $95 \%$ in a population aged 65 years and older [1]. The forecasts for 2050 indicate that the world population aged 60 years and older is expected to total more than 2 billion in 2050 [2]; MM is likely to increase substantially, with the consequent impact on health services $[3,4]$. At the individual level, MM represents reduced physical and mental function, decreased quality of life, low quality of care, increased use of health services, major complications with treatments, and increased mortality [5]. In addition, $\mathrm{MM}$ is common in primary care and treatment management is a major burden for health care professionals [6].

Cardiovascular diseases represent $46 \%$ of noncommunicable disease deaths worldwide [7], and are highly represented in MM. Several recent studies have highlighted hypertension, diabetes, obesity and coronary heart disease as the most frequent MM diagnoses $[1,8,9]$. Furthermore, the concurrence of multiple cardiovascular diseases is an independent predictor of prognosis in patients with established cardiovascular disease [10]. Knowing the cardiovascular disease burden in patients with MM and how these diseases are associated could help clinicians to more effectively search for other diseases when one of them is first diagnosed. It may also contribute to the design of clinical practice guidelines and the development and management of health programs.

The purpose of this study was to describe the burden of cardiovascular diseases in patients with MM by sex and life-stage in a large population sample, evaluate the cardiovascular risk factors (CVRF) and cardiovascular risk (CVR) present a group of patients with $\mathrm{MM}$, and compare these factors with a non-MM group.

\section{Methods}

\section{Data source and study population}

A cross-sectional study of adult residents was conducted in Catalonia, a Mediterranean region of southern Europe with 7,434,632 inhabitants (16\% of the Spanish population, 2010 census). In Catalonia, 358 primary health care teams (PHCTs) comprised of general practitioners (GPs), nurses, social workers and support personnel are assigned by geographical area and responsible for the health care of the population in their areas. The Catalan Health Institute manages 274 PHCTs (76.5\%), serving a population of $4,859,725$ adults; the remaining PHCTs are managed by other providers. Primary care professionals systematically use electronic health records (EHR) to record diagnoses, prescriptions and other clinical information, patient management, and administrative activities. The Catalan Health Institute Information System for the Development of Research in Primary Care (SIDIAP) [11] compiles coded clinical information from the EHR system based on data from its 274 PHCTs. A subset of SIDIAP records meeting the highest quality criteria for clinical data (SIDIAP-Q) includes $40 \%$ of the SIDIAP population $(1,936,443$ patients), attended by the 1,319 GPs assigned to 251 PHCTs whose data recording scored highest in a validated comparison process [12]. SIDIAP has been shown to be highly representative of the general Catalan population in terms of geography, age and sex distribution, according to the official 2010 census [12].

The study sample, was selected from the SIDIAP-Q database, included $1,749,710$ patients aged 19 years or older, assigned to 251 PHCTs during the period of study (1 January- 31 December 2010); 186,733 individuals were excluded because they were younger than 19 years.

\section{Coding of diseases}

International Classification of Diseases (ICD-10) codes were mapped to the International Classification of Primary Care (ICPC-2e-v.4.2, available at: http:// www.kith.no/templates/kith_WebPage___1111.aspx). R codes (symptoms, signs and abnormal clinical and laboratory findings, not elsewhere classified) and $\mathrm{Z}$ codes (factors influencing health status and contact with health services) were excluded, resulting in 686 included codes. Each diagnosis was then classified using O'Halloran criteria for chronic disease [13]: (i) have a duration that has lasted, or is expected to last, at least 6 months; (ii) have a pattern of recurrence or deterioration; (iii) have a poor prognosis and (iv) produce consequences, or sequel, that have an impact on the individual's quality of life $[13,14]$. All results were described by ICPC-2 codes and 146 chronic diseases were included in the analysis.

Cardiovascular morbidity was studied in 24 selected cardiovascular chronic diseases from chapter $\mathrm{K}$ of ICPC2, based on active diagnoses recorded in EHR as of December 31, 2010: Rheumatic fever/heart disease (K71); Neoplasm, cardiovascular (K72); Congenital anomaly, cardiovascular (K73); Ischaemic heart disease with angina (K74); Acute myocardial infarction (K75); Ischaemic heart disease without angina (K76); Heart failure (K77); Atrial fibrillation/flutter (K78); Paroxysmal tachycardia (K79); Cardiac arrhythmia NOS (K80); Heart/arterial murmur NOS (K81); Pulmonary heart disease (K82); Heart valve disease NOS (K83); Heart disease, other (K84); Hypertension, uncomplicated (K86); Hypertension, complicated (K87); Postural hypotension (K88); Transient cerebral ischaemia (K89); Stroke/cerebrovascular accident (K90); Cerebrovascular disease (K91); 
Atherosclerosis/peripheral vascular disease (K92); Pulmonary embolism (K93); Phlebitis/thrombophlebitis (K94) and Varicose veins of leg (K95).

\section{Outcomes and variables}

The main outcome was cardiovascular morbidity burden defined as the coexistence of one or more chronic cardiovascular diseases in patients with MM. MM was defined as the coexistence of 2 or more chronic diseases.

Two study groups were defined, with and without MM. The MM group was further divided into subgroups, constituting a cardiovascular morbidity group (MM-CMG) i.e., MM patients with one or more chronic cardiovascular disease- and non-cardiovascular morbidity group with other chronic diseases (MM-NCMG).

Secondary outcomes included CVR and CVRF profile. CVR was assessed by the REGICOR (Registre Gironí del Cor) score, which evaluates the 10-year risk of a coronary event (angina, myocardial infarct with/without symptoms, fatal or non-fatal), with four categories of severity: low, <5 \%; moderate, 5-9.9 \%; high, 10-14.9\%; and very high, $\geq 15 \%$ [15]. This score is only applicable to individuals aged 35 to 74 years.

Modifiable major CVRFs registered in the EHR and the sum of these major factors were analysed: smoking, hypertension, hypercholesterolaemia and diabetes. Other CVRFs evaluated were: hypertriglyceridaemia, obesity, and alcoholism (in average units of weekly consumption, classified as: low risk consumption [17-28 units in men and 11-17 units in women]; risky consumption [>28 and $>17$, respectively]) $[16,17]$. Five additional variables were considered in the analysis: sex (female/male), age (years), age groups (19-24, 25-44, 45-64, 65-79, and 80 + years), number of chronic diseases and setting (urban/ rural). Physical examinations yielded values for body mass index $\left(\mathrm{BMI}, \mathrm{kg} / \mathrm{cm}^{2}\right)$ and blood pressure $(\mathrm{mm} \mathrm{Hg})$ and included laboratory tests: glycated haemoglobin (\%), creatinine $(\mathrm{mg} / \mathrm{dl})$, uric acid $(\mathrm{mg} / \mathrm{dl})$, total cholesterol $(\mathrm{mg} / \mathrm{dl})$ and triglycerides $(\mathrm{mg} / \mathrm{dl})$, along with glomerular filtration rate $<60 \mathrm{ml} / \mathrm{min} / 1.73 \mathrm{~m}^{2}$ to determine a decrease in renal function [18].

\section{Statistical analysis}

Descriptive statistics were used to summarize overall information. Categorical variables were expressed as frequencies (percentage) and continuous as mean (Standard deviation, SD) or median (interquartile range, IQR). The MM crude prevalence and $95 \%$ confidence intervals $(\mathrm{CI})$ were calculated. The differences between MM and non-MM groups were tested using Student t, Mann-Whitney or Chi-square for unadjusted comparison, as appropriate. The crude prevalence (95\% CI) of MM-CMG was calculated. Prevalence estimates of each $\mathrm{CV}$ chronic condition and $95 \% \mathrm{CI}$ were obtained. The
$95 \%$ CI for the prevalences was calculated using the continuity-corrected Wilson score interval.

For comparison of MM-CMG, MM-NCMG and nonMM groups, ANOVA, Kruskal-Wallis or Chi square tests were used as appropriate. To determine the most prevalent MM patterns in MM-CMG, all possible combinations of each $\mathrm{CV}$ disease, one and two chronic conditions and their frequencies were calculated for each sex and age group. CVR score (REGICOR) distribution was compared within the three groups studied..We assumed that missing data were missing completely at random (MCAR), and so we performed a complete case analysis to handle missing data. We had sufficient power for our analysis, even though we lost part of our data set.

Spearman correlation between number of chronic diseases and chronic cardiovascular diseases and age was assessed.

All statistical tests were two-sided at the $5 \%$ significance level. The analyses were performed using SPSS for Windows, version 18 and $\mathrm{R}$ version 3.2.3.

\section{Results}

A total of $1,749,710$ individuals $\geq 19$ years old were included in the study (Fig. 1). The mean age of the sample was 47.4 years (SD: 17.8) and $50.7 \%$ were women. Nearly half of the total cohort (46.8 \%, 95 \% CI: $46.7-$ 48.1) met MM criteria. Descriptive characteristics of patients in the MM group (including both the MM-CMG and MM-NCMG) and the non-MM group are shown in Table 1. For all variables, significant differences were found between the three study groups (Table 1).

MM prevalence increased with age group (19-24, 25$44,45-64,65-79$, and 80 or more years), as follows: 15.9 \% (95 \% CI: 15.7-16.1), 24.3 \% (95 \% CI: 24.2-24.4), 58.7 \% (95 \% CI:58.6-58.9), 88.7 \% (95 \% CI: 88.6-88.8), and $92.1 \%$ (95\% CI: 91.9-92.2), respectively ( $p<0.001)$.

The total MM population showed significantly higher prevalence $(p<0.001)$ than the non-MM group for three diagnsoses: hypertension (41.1\% vs. $1.8 \%$ ), hypercholesterolaemia (40.4 \% vs. $3.2 \%)$ and diabetes mellitus (15.6 \% vs. $0.5 \%$ ). Except for smoking (23.1 \% vs. $39.0 \%$, $p<0.001)$, the median number of CVRFs in the MM population was 4 (IQR: $2 ; 6)$ vs. 2 (IQR: $1 ; 4)$ in the nonMM group $(p<0.001)$. The median of major CVRFs in patients with $M M$ was 1 (IQR: $1 ; 2)$ and 0 (IQR: $0 ; 1)$ for the non-MM group $(p<0.001)$ (Table 2$)$.

The MM-CMG patients constituted $54.1 \%$ (95 \% CI: 54.0-54.2) of the total MM population (Fig. 1). MMCMG also increased with age group (19-24, 25-44, 45$64,65-79$, and 80 or more years), as follows: $1.6 \%$ (95 \% CI: 1.5-1.6), $5.0 \%$ (95 \% CI: 5.0-5.1), $29.4 \%$ (95 \% CI: 29.3-29.5), $68.9 \%$ (95 \% CI: 68.8-69.1), and $81.6 \%$ (95 \% CI: 81.6-81.4), respectively $(p<0.001)$. The 


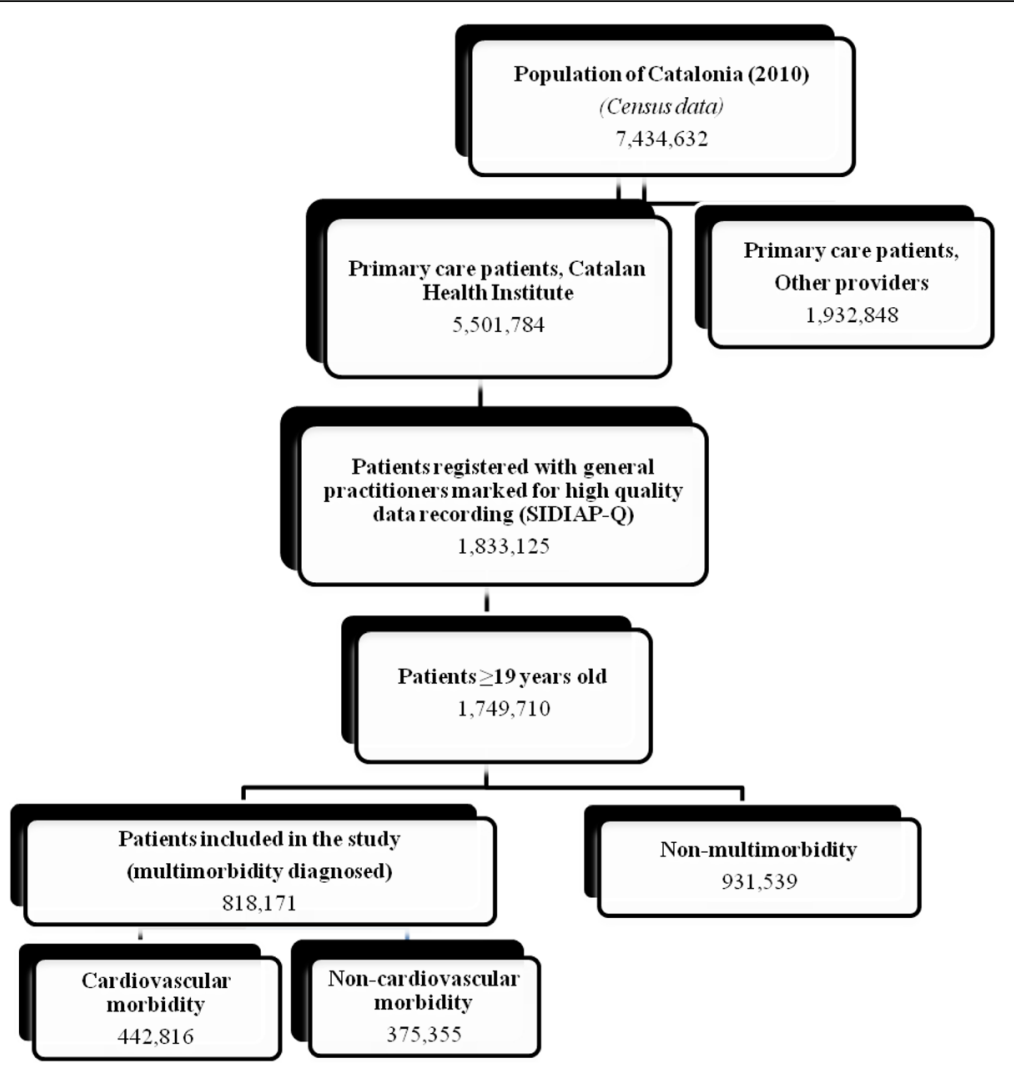

Fig. 1 Sampling framework

four most prevalent cardiovascular diseases were uncomplicated hypertension (75.3\%), varicose veins of the leg $(20.6 \%)$, other heart disease (10.5\%), and atrial fibrillation/flutter (6.7 \%) (Fig. 2). In this group, $38.2 \%$ had more than one cardiovascular disease. Among those patients, the most prevalent combinations were the duet of uncomplicated hypertension \& lipid disorder (38.8\%, $95 \%$ CI: 38.7-39.0) and the triplet of uncomplicated hypertension \& lipid disorder \& non-insulin-dependent diabetes (11.3\%, $95 \%$ CI: 11.2-11.4). By age group, the distribution of duets was uncomplicated hypertension \& lipid disorder in all MM-CMG patients aged 45-80 years and also in younger men, aged 25-44 years; varicose veins of the leg \& anxiety disorder/anxiety in women aged 19-44 years and uncomplicated hypertension \& obesity in the youngest men, aged 19-24 (Table 3). The most prevalent triplets are shown in Table 4.

Significant differences were found in CVR scores between the MM-CMG, MM-NCMG and non-MM groups. Both MM groups had a higher CVR than the non-MM group (Fig. 3).

The correlations between number of chronic diseases and age was $0.60 \quad(p<0.001)$ and between number of chronic cardiovascular diseases and age was $0.57(p 0.001)$.

\section{Discussion}

\section{Main findings}

Cardiovascular diseases accounted for more than $50 \%$ of the burden of chronic disease in patients with MM patients, in part due to a high prevalence of hypertension. Both CVRFs and CVR were increased in patients with MM, compared to the non-MM population. Hypertension, diabetes and dyslipidaemia constituted the most prevalent cardiovascular-endocrine-metabolic pattern.

\section{Strengths and limitations}

The major strength of this study was the analysis of a large, high-quality database of primary-care EHR that has been shown to be representative of a much larger population. Other studies have shown that more accurate conclusions can be drawn from EHR data than from survey-based datasets [19-21]. A number of potential limitations must be taken into account as well. First, chronic diseases could be underreported, which would affect the the observed MM prevalence. For example, $29 \%$ of the Catalan population, especially people with higher socioeconomic status, buys private health insurance to supplement the universal health care services, which could affect population prevalence data. Other variables, such as smoking habit, are under-registered in 
Table 1 Descriptive characteristics of patients by multimorbidity or non-multimorbidity group (1,749,710 adult patients, Catalonia, 2010)

\begin{tabular}{|c|c|c|c|c|}
\hline \multirow[b]{3}{*}{ Variables } & \multicolumn{2}{|c|}{ Multimorbidity group } & \multirow{3}{*}{$\begin{array}{l}\text { Non-multimorbidity } \\
\text { group } \\
(n=931,539)\end{array}$} & \multirow{3}{*}{$\begin{array}{l}\text { Total } \\
\\
(N=1,749,710\end{array}$} \\
\hline & \multirow{2}{*}{$\begin{array}{l}\text { Cardiovascular } \\
\text { morbidity } \\
(n=442,816)\end{array}$} & \multirow{2}{*}{$\begin{array}{l}\text { Non-cardiovascular } \\
\text { morbidity } \\
(n=375,355)\end{array}$} & & \\
\hline & & & & \\
\hline Age (years) & $65.7(14.6)$ & $48.2(15.4)$ & $38.3(12.7)$ & $47.4(17.8)$ \\
\hline Sex (\% females) & 56.1 & 57.5 & 45.5 & 50.7 \\
\hline Number of chronic diseases (median, IQR) & $5.0(3.0 ; 7.0)$ & $3.0(2.0 ; 4.0)$ & $0.0(0.0 ; 1.0)$ & $1.0(0.0 ; 3.0)$ \\
\hline Visits (median, IQR) & $10.0(6.0 ; 18.0)$ & $6.0(3.0 ; 11.0)$ & $1.0(0.0 ; 4.0)$ & $4.0(1.0 ; 9.0)$ \\
\hline Urban setting (\%) & 83.4 & 84.0 & 84.2 & 84.0 \\
\hline \multicolumn{5}{|l|}{ Physical examination } \\
\hline $\mathrm{BMI}\left(\mathrm{kg} / \mathrm{cm}^{2}\right)(n=509,368)$ & $29.7(5.1)$ & $28.0(5.4)$ & $26.0(4.7)$ & $28.3(5.3)$ \\
\hline Systolic BP (mm Hg) $(n=773,446)$ & $135.2(16.2)$ & $126.6(14.6)$ & $123.8(14.9)$ & $129.7(16.3)$ \\
\hline Diastolic BP $(\mathrm{mm} \mathrm{Hg})(n=774,139)$ & $76.7(10.3)$ & $76.0(9.6)$ & $74.5(10.1)$ & $75.9(10.1)$ \\
\hline \multicolumn{5}{|l|}{ Laboratory tests } \\
\hline Glycated haemoglobin (\%)(SD) $(n=208,581)$ & $6.6(1.3)$ & $6.3(1.5)$ & $5.7(1.2)$ & $6.4(1.4)$ \\
\hline Creatinine $(\mathrm{mg} / \mathrm{dl})(\mathrm{SD})(n=738,083)$ & $0.9(0.4)$ & $0.8(0.2)$ & $0.8(0.2)$ & $0.9(0.3)$ \\
\hline Uric acid (mg/dl) (SD) $(n=475,334)$ & $5.5(1.6)$ & $4.8(1.4)$ & $4.8(1.4)$ & $5.2(1.5)$ \\
\hline Total cholesterol $(\mathrm{mg} / \mathrm{dl})(\mathrm{SD})(n=745,010)$ & $202.0(39.7)$ & $209.0(40.6)$ & $194.3(38.4)$ & $201.8(40.0)$ \\
\hline Triglycerides (mg/dl) (SD) $(n=190,159)$ & $132.9(82.9)$ & $129(98.7)$ & $113.3(75.7)$ & $127.5(86.4)$ \\
\hline Glomerular filtration rate < $60 \%(\%)(n=544,438)$ & 17.6 & 3.2 & 1.3 & 9.2 \\
\hline
\end{tabular}

$p$-values overall between multimorbidity and non-multimorbidity groups were significant for all variables $(p<0.001)$

Table 2 Distribution of cardiovascular risk factors by multimorbidity and non-multimorbidity groups (1,749,710 adult patients, Catalonia, 2010)

\begin{tabular}{|c|c|c|c|c|}
\hline & \multicolumn{2}{|c|}{ Multimorbidity group } & \multirow[t]{2}{*}{ Non-multimorbidity group } & \multirow[t]{2}{*}{ Total } \\
\hline & $\begin{array}{l}\text { Cardiovascular } \\
\text { morbidity }\end{array}$ & $\begin{array}{l}\text { Non-cardiovascular } \\
\text { morbidity }\end{array}$ & & \\
\hline & $(n=442,816)$ & $(n=375,355)$ & $(n=931,539)$ & $(N=1,749,710)$ \\
\hline \multicolumn{5}{|l|}{ Major modifiable cardiovascular risk factors } \\
\hline Smoking $(n=1,329,331)$ & 15.1 & 33.3 & 39.0 & 29.9 \\
\hline Hypertension $(n=1,749,710)$ & 75.9 & 0.0 & 1.8 & 20.2 \\
\hline Hypercholesterolemia $(n=1,749,710)$ & 47.7 & 31.8 & 3.2 & 20.6 \\
\hline Diabetes $(n=1,749,710)$ & 22.8 & 7.1 & 0.5 & 7.6 \\
\hline \multicolumn{5}{|l|}{ Other factors } \\
\hline Hypertriglyceridemia $(n=190,159)$ & 27.8 & 24.9 & 18.2 & 24.9 \\
\hline Obesity $(n=1,749,710)$ & 24.5 & 15.5 & 1.8 & 10.5 \\
\hline Alcoholism $(n=577,213)$ & 28.2 & 29.8 & 32.4 & 29.9 \\
\hline Sum of major factors (median, [IQR]) $(n=1,329,331)$ & $2[1 ; 2]$ & $1[0 ; 1]$ & $0[0 ; 1]$ & $1[0 ; 1]$ \\
\hline Cardiovascular risk score (REGICOR) (median, [IQR]) $(n=264,773)^{a}$ & $4[3 ; 7]$ & $3[2 ; 5]$ & $2[1 ; 4]$ & $4[2 ; 6]$ \\
\hline \multicolumn{5}{|l|}{ Cardiovascular risk score (REGICOR) $(n=264,773)^{\mathrm{a}}$} \\
\hline Low & 60.6 & 74.6 & 84.5 & 67.4 \\
\hline Moderate & 30.1 & 20.5 & 13.4 & 25.4 \\
\hline High & 6.9 & 3.8 & 1.7 & 5.4 \\
\hline Very high & 2.4 & 1.1 & 0.4 & 1.8 \\
\hline
\end{tabular}




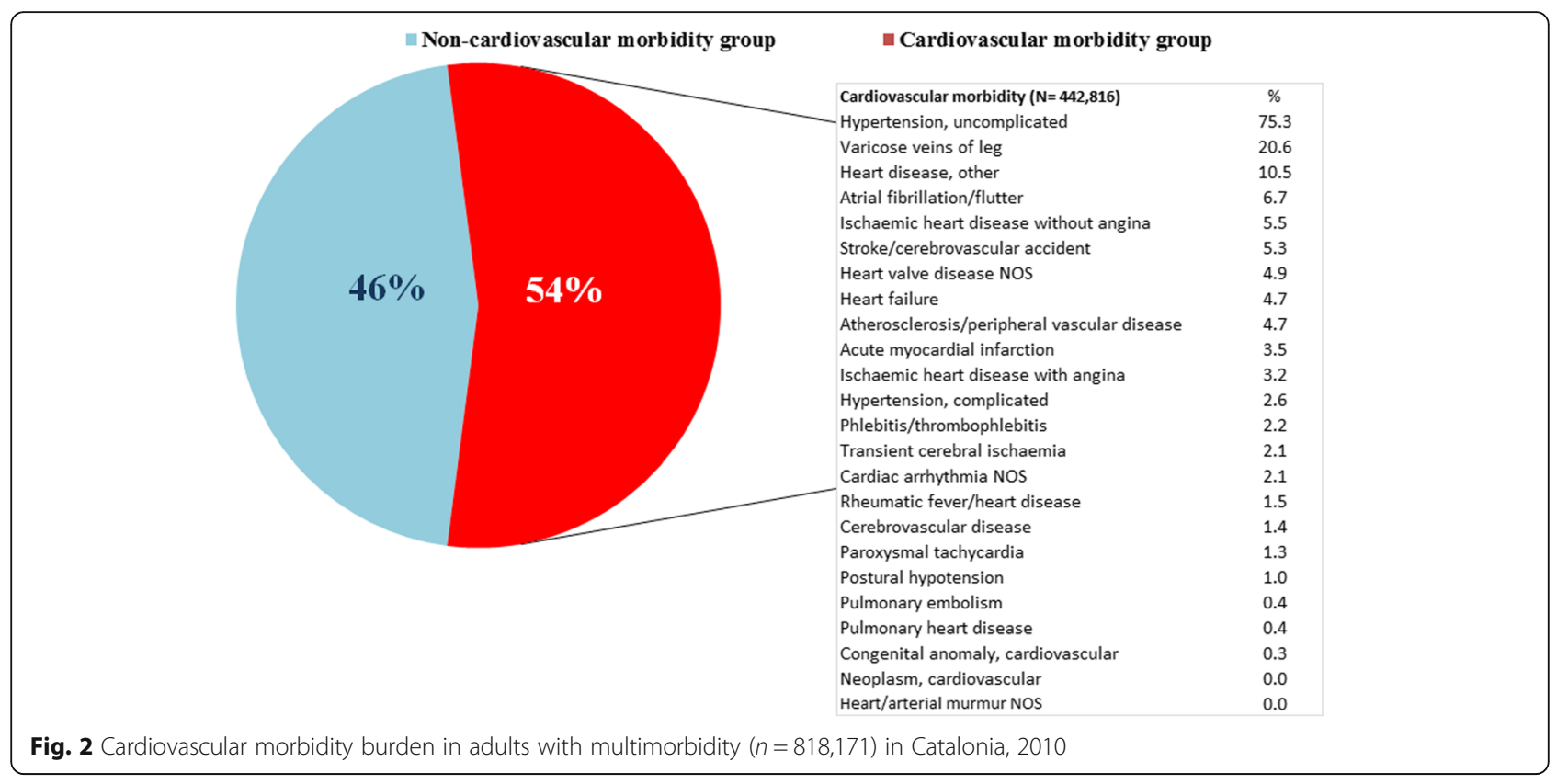

the EHR (notably in the MM-CVG, exactly the group that should be addressed with precision on this variable), and this may modify the final results.

On the other hand, there could be an overrepresentation of chronic diagnoses (e.g., hypertension, diabetes, hyperlipidaemia, etc.) that are included in the goal/incentive contracts of Catalan PHCTs. Another aspect is the definition and counting of the chronic conditions included, which could modify the results as showen in previous studies $[22,23]$. Finally, residual confounding cannot be completely excluded; certain epidemiological factors or other health determinants were not considered in this study, such as environmental factors.

\section{Comparison with existing literature}

Very few studies have evaluated the cardiovascular burden in MM. In a sample of 73,254 persons of all ages, Landwehr et al. observed high comorbidity within a group of 30 cardiovascular disorders [24]. Another more recent study of cardiovascular diseases in patients with MM aged $\geq 18$ years in a primary care setting showed that almost $60 \%$ of the patients included had one cardiovascular disease and $34 \%$ had two of them. Their study focused on ethnic differences and did not provide data about combinations of diseases or MM patterns by age groups [8]. Other studies have shown a high impact of cardiovascular diseases in middle-age, resulting in increased mortality risk $[25,26]$.

In addition, the prevalence of $\mathrm{MM}$ and the main patterns described in different studies focused in Spain may vary from other European countries. The number of chronic conditions included, age ranges of the study population and the definition of $\mathrm{MM}$ are the major explanations for these differences $[9,27]$. Different Spanish regions also have differences in MM prevalences: 47 \% (ages 19+) in Catalonia [28], 37 \% (15+) in Aragon [9], and $24 \%$ (all ages) in Basque Country [28], all from EHR analysis, and $69 \%(18+)$ in a representative survey of the Spanish population [29]. These differences may be due to the methodology used in these studies or to the effects of socioeconomic inequalities between territorial regions of Spain [30]. Nonetheless, cardiovascular diseases are present in the main MM patterns reported in most countries. In a recent systematic review that included observational studies performed in primary care settings, hypertension and osteoarthritis were the most frequent combination, followed by other combinations that included cardiovascular diseases [1]. Similarly, in our study the most frequent combination was uncomplicated hypertension \& lipid disorder.

Hypertension is the most prevalent disease related to medical consultation by those aged 65 and older [31]. The addition to hypertension of other cardiovascular diseases has been associated with increased morbidity and consequently with increased costs [32]. The heavy burden on health services indicates that cardiovascular morbidity requires major efforts in to prevent these diseases and especially a comprehensive approach to CVR prevention and reduction [33]. Although data about cardiovascular comorbidity are readily available, particularly with regard to a specific index disease (hypertension, coronary heart disease, heart failure, etc.) [34-36], published data on cardiovascular diseases in MM are scarce. In the primary health care setting, however, the MM approach has been recommended as more suitable than focusing on one specific disease $[3,37]$. 
Table 3 Most prevalent comorbidities associated with cardiovascular diseases in multimorbid patients with cardiovascular morbidity, by age group and sex (442,816 adult patients, Catalonia, 2010)

\begin{tabular}{|c|c|c|c|c|c|c|c|c|c|c|}
\hline \multirow[t]{2}{*}{$\begin{array}{l}\text { Age Groups } \\
\text { (years) }\end{array}$} & \multicolumn{5}{|l|}{$\begin{array}{l}\text { Female } \\
n=248,407\end{array}$} & \multicolumn{5}{|l|}{$\begin{array}{l}\text { Male } \\
n=194,409\end{array}$} \\
\hline & Cardiovascular index disease & Associated disease 1 & $n$ & $\%$ & $95 \% \mathrm{Cl}$ & Cardiovascular index disease & Associated disease 1 & $n$ & $\%$ & $95 \% \mathrm{Cl}$ \\
\hline \multirow{5}{*}{$\begin{array}{l}19-24 \\
n=2,152\end{array}$} & Varicose veins of leg & Anxiety disorder/anxiety state & 92 & 7.1 & $(5.8-8.7)$ & Hypertension, uncomplicated & Obesity & 78 & 9.0 & $(7.2-11.2)$ \\
\hline & Postural hypotension & Anxiety disorder/anxiety state & 83 & 6.4 & $(5.2-8.0)$ & Heart disease, other & Acne & 39 & 4.5 & $(3.3-6.2)$ \\
\hline & Hypertension, uncomplicated & Obesity & 35 & 2.7 & $(1.9-3.8)$ & Postural hypotension & Acne & 16 & 1.8 & $(1.1-3.1)$ \\
\hline & Paroxysmal tachycardia & Anxiety disorder/anxiety state & 31 & 2.4 & $(1.7-3-4)$ & Varicose veins of leg & Acquired deformity of spine & 16 & 1.8 & $(0.8-2.6)$ \\
\hline & Heart disease, other & Anxiety disorder/anxiety state & 24 & 1.9 & $(1.2-2.8)$ & Congenital anomaly cardiovascular & Acne & 13 & 1.5 & $(0.8-2.6)$ \\
\hline \multirow{5}{*}{$\begin{array}{l}25-44 \\
n=37,308\end{array}$} & Varicose veins of leg & Anxiety disorder/anxiety state & 2,794 & 13.0 & $(12.6-13.5)$ & Hypertension, uncomplicated & Lipid disorder & 3,425 & 21.6 & $(21.0-22.3)$ \\
\hline & Hypertension, uncomplicated & Obesity & 2,125 & 9.9 & $(9.5-10.3)$ & Varicose veins of leg & Lipid disorder & 476 & 3.0 & $(2.7-3.3)$ \\
\hline & Postural hypotension & Anxiety disorder/anxiety state & 527 & 2.5 & $(2.3-2.7)$ & Heart disease, other & Lipid disorder & 451 & 2.8 & $(2.6-3.1)$ \\
\hline & Heart disease, other & Anxiety disorder/anxiety state & 253 & 1.2 & $(1.0-1.3)$ & Hypertension, complicated & Hypertension, uncomplicated & 201 & 1.3 & $(1.1-1.5)$ \\
\hline & Paroxysmal tachycardia & Anxiety disorder/anxiety state & 215 & 1.0 & $(0.9-1.1)$ & Acute myocardial infarction & Lipid disorder & 159 & 1.0 & $(0.9-1.2)$ \\
\hline \multirow{5}{*}{$\begin{array}{l}45-64 \\
n=154,049\end{array}$} & Hypertension, uncomplicated & Lipid disorder & 25,930 & 32.5 & $(32.2-32.9)$ & Hypertension, uncomplicated & Lipid disorder & 30,975 & 41.7 & $(41.3-42.0)$ \\
\hline & Varicose veins of leg & Lipid disorder & 10,339 & 13.0 & $(12.7-13.2)$ & Heart disease, other & Hypertension, uncomplicated & 4,186 & 5.6 & $(5.5-5.8)$ \\
\hline & Heart disease, other & Hypertension, uncomplicated & 2,057 & 2.6 & $(2.5-2.7)$ & Ischaemic heart disease w/o angina & Lipid disorder & 2,992 & 4.0 & $(3.9-4.2)$ \\
\hline & Heart valve disease NOS & Hypertension, uncomplicated & 1,131 & 1.4 & $(1.3-1.5)$ & Varicose veins of leg & Lipid disorder & 2,745 & 3.7 & $(3.6-3.8)$ \\
\hline & Stroke/cerebrovascular accident & Hypertension, uncomplicated & 895 & 1.1 & $(1.1-1.2)$ & Acute myocardial infarction & Lipid disorder & 2,582 & 3.5 & $(3.3-3.6)$ \\
\hline \multirow{5}{*}{$\begin{array}{l}65-79 \\
n=168,340\end{array}$} & Hypertension, uncomplicated & Lipid disorder & 46,236 & 49.5 & $(49.2-49.8)$ & Hypertension, uncomplicated & Lipid disorder & 32,003 & 42.7 & $(42.3-43.1)$ \\
\hline & Varicose veins of leg & Hypertension, uncomplicated & 16,214 & 17.4 & $(17.1-17.6)$ & Heart disease, other & Hypertension, uncomplicated & 8,156 & 10.9 & $(10.7-11.1)$ \\
\hline & Heart disease, other & Hypertension, uncomplicated & 7,075 & 7.6 & $(7.4-7.7)$ & Ischaemic heart disease w/o angina & Hypertension, uncomplicated & 5,297 & 7.1 & $6.9-7.3)$ \\
\hline & Atrial fibrillation/flutter & Hypertension, uncomplicated & 4,739 & 5.1 & $(4.9-5.2)$ & Atrial fibrillation/flutter & Hypertension, uncomplicated & 4,765 & 6.4 & $(6.2-6.5)$ \\
\hline & Heart valve disease NOS & Hypertension, uncomplicated & 4,164 & 4.5 & $(4.3-4.6)$ & Stroke/cerebrovascular accident & Hypertension, uncomplicated & 4,522 & 6.0 & $(5.9-6.2)$ \\
\hline \multirow{5}{*}{$\begin{array}{l}80+ \\
n=80,967\end{array}$} & Hypertension, uncomplicated & Lipid disorder & 22,984 & 43.7 & $(43.3-44.2)$ & Hypertension, uncomplicated & Lipid disorder & 9,115 & 32.1 & $(31.5-32.6)$ \\
\hline & Varicose veins of leg & Hypertension, uncomplicated & 8,923 & 17.0 & $(16.7-17.3)$ & Heart disease, other & Hypertension, uncomplicated & 4,244 & 14.9 & $(14.5-15.4)$ \\
\hline & Atrial fibrillation/flutter & Hypertension, uncomplicated & 6,404 & 12.2 & $(11.9-12.5)$ & Atrial fibrillation/flutter & Hypertension, uncomplicated & 3,600 & 12.7 & $(12.3-13.1)$ \\
\hline & Heart disease, other & Hypertension, uncomplicated & 6,022 & 11.5 & $(11.2-11.7)$ & Stroke/cerebrovascular accident & Hypertension, uncomplicated & 2,651 & 9.3 & $(9.0-9.7)$ \\
\hline & Heart failure & Hypertension, uncomplicated & 5,814 & 11.1 & $(10.8-11.3)$ & Heart failure & Hypertension, uncomplicated & 2,579 & 9.1 & $(8.7-9.4)$ \\
\hline
\end{tabular}


Table 4 Two most prevalent comorbidity diseases associated with cardiovascular diseases in multimorbid patients with cardiovascular morbidity, by age group and sex (442,816 adult patients, Catalonia, 2010)

\begin{tabular}{|c|c|c|c|c|c|c|c|c|c|c|c|c|}
\hline \multirow[t]{2}{*}{$\begin{array}{l}\text { Age Groups } \\
\text { (years) }\end{array}$} & \multicolumn{6}{|l|}{$\begin{array}{l}\text { Women } \\
n=248,407\end{array}$} & \multicolumn{6}{|l|}{$\begin{array}{l}\text { Men } \\
n=194,409\end{array}$} \\
\hline & $\begin{array}{l}\text { Index } \\
\text { cardiovascular } \\
\text { disease }\end{array}$ & $\begin{array}{l}\text { Associated } \\
\text { disease } 1\end{array}$ & $\begin{array}{l}\text { Associated } \\
\text { disease } 2\end{array}$ & $\mathrm{n}$ & $\%$ & $95 \% \mathrm{Cl}$ & $\begin{array}{l}\text { Index cardiovascular } \\
\text { disease }\end{array}$ & $\begin{array}{l}\text { Associated } \\
\text { disease } 1\end{array}$ & $\begin{array}{l}\text { Associated } \\
\text { disease } 2\end{array}$ & $\mathrm{n}$ & $\%$ & $95 \% \mathrm{Cl}$ \\
\hline \multirow[t]{5}{*}{$\begin{array}{l}19-24 \\
n=2,152\end{array}$} & $\begin{array}{l}\text { Postural } \\
\text { hypotension }\end{array}$ & Acne & $\begin{array}{l}\text { Anxiety disorder/ } \\
\text { anxiety state }\end{array}$ & 14 & 1.1 & $(0.6-1.9)$ & $\begin{array}{l}\text { Hypertension, } \\
\text { uncomplicated }\end{array}$ & Obesity & Acne & 8 & 0.9 & $(0.4-1.9)$ \\
\hline & $\begin{array}{l}\text { Varicose veins of } \\
\text { leg }\end{array}$ & Acne & $\begin{array}{l}\text { Anxiety disorder/ } \\
\text { anxiety state }\end{array}$ & 13 & 1.0 & $(0.6-1.8)$ & Heart disease, other & $\begin{array}{l}\text { Dermatitis/ } \\
\text { atopic eczema }\end{array}$ & Asthma & 6 & 0.7 & $(0.3-1.6)$ \\
\hline & $\begin{array}{l}\text { Hypertension, } \\
\text { uncomplicated }\end{array}$ & $\begin{array}{l}\text { Endocrine/metabolic/ } \\
\text { nutritional disorder, other }\end{array}$ & Obesity & 9 & 0.7 & $(0.3-1.4)$ & $\begin{array}{l}\text { Congenital anomaly } \\
\text { cardiovascular }\end{array}$ & Asthma & $\begin{array}{l}\text { Heart valve disease } \\
\text { NOS }\end{array}$ & 3 & 0.3 & $(0.1-1.1)$ \\
\hline & $\begin{array}{l}\text { Heart valve } \\
\text { disease NOS }\end{array}$ & Acne & Acne & 5 & 0.4 & $(0.1-1.0)$ & $\begin{array}{l}\text { Cardiac arrhythmia } \\
\text { NOS }\end{array}$ & Acne & $\begin{array}{l}\text { Musculoskeletal } \\
\text { disease, other }\end{array}$ & 3 & 0.3 & $(0.1-1.1)$ \\
\hline & $\begin{array}{l}\text { Paroxysmal } \\
\text { tachycardia }\end{array}$ & Depressive disorder & $\begin{array}{l}\text { Anxiety disorder/ } \\
\text { anxiety state }\end{array}$ & 4 & 0.3 & $(0.1-0.9)$ & $\begin{array}{l}\text { Heart valve disease } \\
\text { NOS }\end{array}$ & Asthma & $\begin{array}{l}\text { Congenital anomaly, } \\
\text { cardiovascular }\end{array}$ & 3 & 0.3 & $(0.1-1.1)$ \\
\hline \multirow[t]{5}{*}{$\begin{array}{l}25-44 \\
n=37,308\end{array}$} & $\begin{array}{l}\text { Hypertension, } \\
\text { uncomplicated }\end{array}$ & Lipid disorder & Obesity & 538 & 2.5 & $(2.3-2.7)$ & $\begin{array}{l}\text { Hypertension, } \\
\text { uncomplicated }\end{array}$ & Lipid disorder & Obesity & 1,076 & 6.8 & $(6.4-7.2)$ \\
\hline & $\begin{array}{l}\text { Varicose veins of } \\
\text { leg }\end{array}$ & Depressive disorder & $\begin{array}{l}\text { Anxiety disorder/ } \\
\text { anxiety state }\end{array}$ & 491 & 2.3 & $(2.1-2.5)$ & Heart disease, other & Lipid disorder & $\begin{array}{l}\text { Hypertension, } \\
\text { uncomplicated }\end{array}$ & 138 & 0.9 & $(0.7-1.0)$ \\
\hline & $\begin{array}{l}\text { Postural } \\
\text { hypotension }\end{array}$ & Depressive disorder & $\begin{array}{l}\text { Anxiety disorder/ } \\
\text { anxiety state }\end{array}$ & 102 & 0.5 & $(0.4-0.6)$ & Varicose veins of leg & Lipid disorder & Obesity & 105 & 0.7 & $(0.5-0.8)$ \\
\hline & $\begin{array}{l}\text { Phlebitis/thrombo- } \\
\text { phlebitis }\end{array}$ & Obesity & $\begin{array}{l}\text { Varicose veins of } \\
\text { leg }\end{array}$ & 59 & 0.3 & $(0.2-0.4)$ & $\begin{array}{l}\text { Hypertension, } \\
\text { complicated }\end{array}$ & Lipid disorder & $\begin{array}{l}\text { Hypertension, } \\
\text { uncomplicated }\end{array}$ & 66 & 0.4 & $(0.3-0.5$ \\
\hline & $\begin{array}{l}\text { Heart disease, } \\
\text { other }\end{array}$ & Depressive disorder & $\begin{array}{l}\text { Anxiety disorder/ } \\
\text { anxiety state }\end{array}$ & 46 & 0.2 & $(0.2-0-3)$ & $\begin{array}{l}\text { Acute myocardial } \\
\text { infarction }\end{array}$ & Lipid disorder & $\begin{array}{l}\text { Hypertension, } \\
\text { uncomplicated }\end{array}$ & 60 & 0.4 & $(0.3-0.5$ \\
\hline \multirow[t]{5}{*}{$\begin{array}{l}45-64 \\
n=154,049\end{array}$} & $\begin{array}{l}\text { Hypertension, } \\
\text { uncomplicated }\end{array}$ & Lipid disorder & Obesity & 9,134 & 11.5 & $(11.2-11.7)$ & $\begin{array}{l}\text { Hypertension, } \\
\text { uncomplicated }\end{array}$ & Lipid disorder & $\begin{array}{l}\text { Diabetes, non-insulin } \\
\text { dependent }\end{array}$ & 8,631 & 11.6 & $(11.4-11.8)$ \\
\hline & $\begin{array}{l}\text { Varicose veins of } \\
\text { leg }\end{array}$ & Lipid disorder & $\begin{array}{l}\text { Hypertension, } \\
\text { uncomplicated }\end{array}$ & 3,761 & 4.7 & $(4.6-4.9)$ & Heart disease, other & Lipid disorder & $\begin{array}{l}\text { Hypertension, } \\
\text { uncomplicated }\end{array}$ & 2,275 & 3.1 & $(2.9-3.2)$ \\
\hline & $\begin{array}{l}\text { Heart disease, } \\
\text { other }\end{array}$ & Lipid disorder & $\begin{array}{l}\text { Hypertension, } \\
\text { uncomplicated }\end{array}$ & 1,081 & 1.4 & $(1.3-1.4)$ & $\begin{array}{l}\text { Ischaemic heart } \\
\text { disease w/o angina }\end{array}$ & Lipid disorder & $\begin{array}{l}\text { Hypertension, } \\
\text { uncomplicated }\end{array}$ & 1,744 & 2.3 & $(2.2-2.5)$ \\
\hline & $\begin{array}{l}\text { Heart valve } \\
\text { disease NOS }\end{array}$ & Lipid disorder & $\begin{array}{l}\text { Hypertension, } \\
\text { uncomplicated }\end{array}$ & 578 & 0.7 & $(0.7-0-8)$ & $\begin{array}{l}\text { Acute myocardial } \\
\text { infarction }\end{array}$ & Lipid disorder & $\begin{array}{l}\text { Hypertension, } \\
\text { uncomplicated }\end{array}$ & 1,361 & 1.8 & $(1.7-1.9)$ \\
\hline & $\begin{array}{l}\text { Stroke/ } \\
\text { cerebrovascular } \\
\text { accident }\end{array}$ & Lipid disorder & $\begin{array}{l}\text { Hypertension, } \\
\text { uncomplicated }\end{array}$ & 500 & 0.6 & $(0.6-0.7)$ & Atherosclerosis/PVD & Lipid disorder & $\begin{array}{l}\text { Hypertension, } \\
\text { uncomplicated }\end{array}$ & 1,275 & 1.7 & $(1.6-1.8)$ \\
\hline
\end{tabular}


Table 4 Two most prevalent comorbidity diseases associated with cardiovascular diseases in multimorbid patients with cardiovascular morbidity, by age group and sex (442,816 adult patients, Catalonia, 2010) (Continued)

\begin{tabular}{|c|c|c|c|c|c|c|c|c|c|c|c|c|}
\hline \multirow[t]{5}{*}{$\begin{array}{l}65-79 \\
n=168,340\end{array}$} & $\begin{array}{l}\text { Hypertension, } \\
\text { uncomplicated }\end{array}$ & Lipid disorder & Obesity & 15,115 & 16.2 & $(15.9-16.4)$ & $\begin{array}{l}\text { Hypertension, } \\
\text { uncomplicated }\end{array}$ & Lipid disorder & $\begin{array}{l}\text { Diabetes, non-insulin } \\
\text { dependent }\end{array}$ & 11,573 & 15.4 & $(15.2-15.7)$ \\
\hline & $\begin{array}{l}\text { Varicose veins of } \\
\text { leg }\end{array}$ & Lipid disorder & $\begin{array}{l}\text { Hypertension, } \\
\text { uncomplicated }\end{array}$ & 9,365 & 10.0 & $(9.8-10.2)$ & Heart disease, other & Lipid disorder & $\begin{array}{l}\text { Hypertension, } \\
\text { uncomplicated }\end{array}$ & 4,389 & 5.9 & $(5.7-6.0)$ \\
\hline & $\begin{array}{l}\text { Heart disease, } \\
\text { other }\end{array}$ & Lipid disorder & $\begin{array}{l}\text { Hypertension, } \\
\text { uncomplicated }\end{array}$ & 4,298 & 4.6 & $(4.5-4.7)$ & $\begin{array}{l}\text { Ischaemic heart } \\
\text { disease w/o angina }\end{array}$ & Lipid disorder & $\begin{array}{l}\text { Hypertension, } \\
\text { uncomplicated }\end{array}$ & 3,346 & 4.5 & $(4.3-4.6)$ \\
\hline & $\begin{array}{l}\text { Heart valve } \\
\text { disease NOS }\end{array}$ & Lipid disorder & $\begin{array}{l}\text { Hypertension, } \\
\text { uncomplicated }\end{array}$ & 2,530 & 2.7 & $(2.6-2.8)$ & Atherosclerosis/PVD & Lipid disorder & $\begin{array}{l}\text { Hypertension, } \\
\text { uncomplicated }\end{array}$ & 2675 & 3.6 & $(3.4-3.7)$ \\
\hline & $\begin{array}{l}\text { Atrial fibrillation/ } \\
\text { flutter }\end{array}$ & Lipid disorder & $\begin{array}{l}\text { Hypertension, } \\
\text { uncomplicated }\end{array}$ & 2,525 & 2.7 & $(2.6-2.8)$ & $\begin{array}{l}\text { Stroke/ } \\
\text { cerebrovascular } \\
\text { accident }\end{array}$ & Lipid disorder & $\begin{array}{l}\text { Hypertension, } \\
\text { uncomplicated }\end{array}$ & 2,557 & 3.4 & $(3.3-3.5)$ \\
\hline \multirow[t]{5}{*}{$\begin{array}{l}80+ \\
n=80,967\end{array}$} & $\begin{array}{l}\text { Hypertension, } \\
\text { uncomplicated }\end{array}$ & Lipid disorder & $\begin{array}{l}\text { Diabetes, non- } \\
\text { insulin dependent }\end{array}$ & 6,769 & 12.9 & $(12.6-13.2)$ & $\begin{array}{l}\text { Hypertension, } \\
\text { uncomplicated }\end{array}$ & $\begin{array}{l}\text { Benign prostatic } \\
\text { hypertrophy }\end{array}$ & Lipid disorder & 3658 & 12.9 & $(12.5-13.3)$ \\
\hline & $\begin{array}{l}\text { Varicose veins of } \\
\text { leg }\end{array}$ & Lipid disorder & $\begin{array}{l}\text { Hypertension, } \\
\text { uncomplicated }\end{array}$ & 4,445 & 8.5 & $(8.2-8.7)$ & Heart disease, other & Lipid disorder & $\begin{array}{l}\text { Hypertension, } \\
\text { uncomplicated }\end{array}$ & 1,823 & 6.4 & $(6.1-6.7)$ \\
\hline & $\begin{array}{l}\text { Heart disease, } \\
\text { other }\end{array}$ & Lipid disorder & $\begin{array}{l}\text { Hypertension, } \\
\text { uncomplicated }\end{array}$ & 3,255 & 6.2 & $(6.0-6.4)$ & $\begin{array}{l}\text { Atrial fibrillation/ } \\
\text { flutter }\end{array}$ & $\begin{array}{l}\text { Benign prostatic } \\
\text { hypertrophy }\end{array}$ & $\begin{array}{l}\text { Hypertension, } \\
\text { uncomplicated }\end{array}$ & 1,466 & 5.2 & $(4.9-5.4)$ \\
\hline & $\begin{array}{l}\text { Atrial fibrillation/ } \\
\text { flutter }\end{array}$ & Lipid disorder & $\begin{array}{l}\text { Hypertension, } \\
\text { uncomplicated }\end{array}$ & 2,900 & 5.5 & $(5.3-5.7)$ & $\begin{array}{l}\text { Ischaemic heart } \\
\text { disease w/o angina }\end{array}$ & Lipid disorder & $\begin{array}{l}\text { Hypertension, } \\
\text { uncomplicated }\end{array}$ & 1,357 & 4.8 & $(4.5-5.0)$ \\
\hline & Heart failure & Lipid disorder & $\begin{array}{l}\text { Hypertension, } \\
\text { uncomplicated }\end{array}$ & 2,776 & 5.3 & $(5.1-5.5)$ & $\begin{array}{l}\text { Stroke/ } \\
\text { cerebrovascular } \\
\text { accident }\end{array}$ & Lipid disorder & $\begin{array}{l}\text { Hypertension, } \\
\text { uncomplicated }\end{array}$ & 1,153 & 4.1 & $(3.8-4.3)$ \\
\hline
\end{tabular}


Cardiovascular risk score

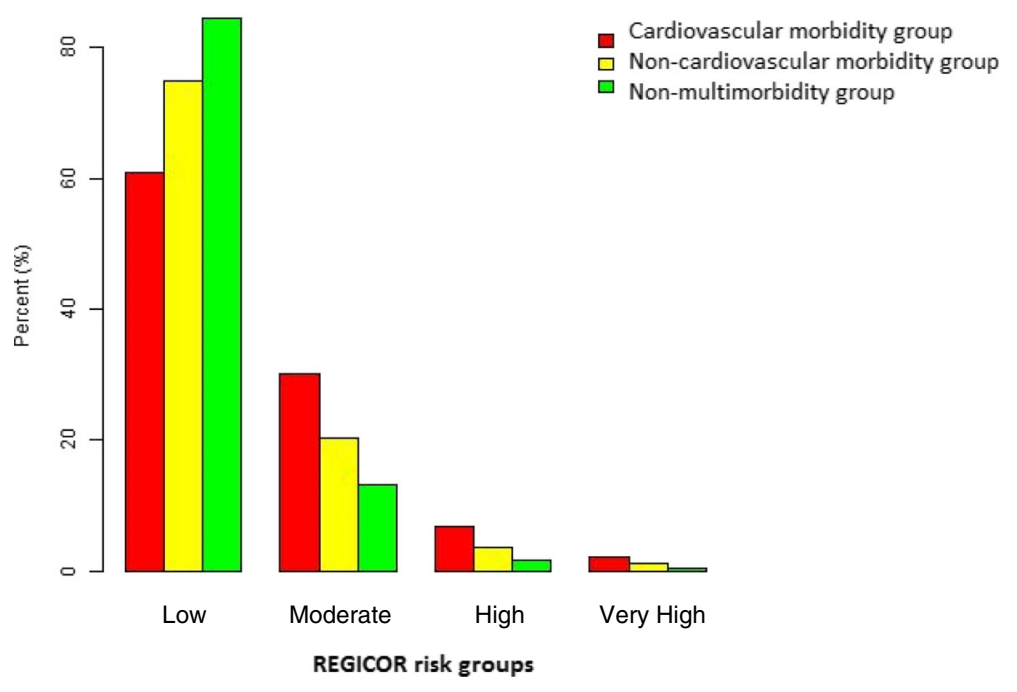

Note: All comparisons between groups had a p-value $<0.001$.

Fig. 3 Cardiovascular risk distribution by multimorbidity or non-multimorbidity group (264,773 adult patients aged 35-74 years, Catalonia, 2010)

A recent literature review evaluated MM studies that specifically analysed cardiovascular diseases, and found that almost all were focused on an index disease (e.g., coronary heart disease or heart failure) or a limited number of cardiovascular diseases [38]. Another very recent study called for practice-based research focused on common dyads and triads in order to translate these results to best-practice guidelines, including disease patterns even when the relationships between them are not fully understood (for example, coexisting depression and CVD) [39]. Our study, evaluated the comorbidity of 24 chronic cardiovascular diseases, focusing the attention on these diseases and providing data on the patterns observed.

\section{Impact of the study}

The findings from this study make several contributions to the current literature. Despite considerable knowledge about the separate impacts of cardiovascular diseases on the burden of chronic diseases, there is a gap in knowledge about their impact on $\mathrm{MM}$ as a whole. The present study quantifies this impact, based on analysis of a large, high-quality database. On the other hand, our data provide more information about the associations between cardiovascular diseases and MM, which may help clinicians to understand the complexity of MM. Our analysis of MM-CMG patterns showed a high prevalence of certain diseases accompanying cardiovascular diseases, such as lipid disorders in men aged 25 years and older and women aged 45 years and older. This could be a useful consideration for clinicians screening for comorbidities when a chronic disease is first diagnosed. Another finding of clinical interest was the association of depressive disorder and anxiety with paroxysmal tachycardia in younger women (19-24 years) and with heart disease in women aged 25-44 years. When the first of these cardiovascular diagnoses is made clinicians may be well advised to explore symptoms in the mental health sphere.

According to the observed presentation of associated diseases in the present study results, future studies could investigate the chronological order of diseases and the interventions in family practice that are most adequate to prevent the accumulation of diseases. Another potential impact of these results would be to introduce the concept of multimorbidity earlier in university degree programs that prepare health professionals, changing the disease-specific focus to a multimorbidity approach. Finally, in the field of health policy, efforts should focus on programs to avoid the accumulation of diseases when one disease appears, introducing guidelines on the management of associated diseases and the use of predictive algorithms that help doctors to identify occult diseases based on probabilistic models.

\section{Future research}

Future research is needed to establish the optimal approaches to prevent or avoid the patient progression from non-MM to diagnsosis of one or more cardiovascular diseases in addition to other chronic but preventable diseases. In this sense, longitudinal studies should be performed to establish the specific moment of disease occurrence, how diseases are related over time and what 
interventions can be useful to prevent the onset of comorbidities in various patient populations.

\section{Conclusions}

Data from a large sample of primary health care records showed that cardiovascular diseases represent more than half of the MM burden. Cardiovascular risk factors and risk profiles were higher in patients with $M M$ than in the non-MM group, mainly due to the high prevalence of hypertension. Hypertension, diabetes and dyslipidaemia constitute the most prevalent cardiovascularendocrine-metabolic pattern.

\section{Abbreviations}

BMI: Body mass index; CVR: Cardiovascular risk; CVRF: Cardiovascular risk factors; EHR: Electronic health records; GP: General practitioners; ICD-10: International classification of diseases; ICPC: International classification of primary care; MM: Multimorbidity; MM-CMG: Multimorbidity- cardiovascular morbidity group; MM-NCMG: IDIAP Jordi Gol, Institut Universitari d'Investigació en Atenció Primària Jordi Gol; PHCT: Primary health care teams; REGICOR: Registre Gironi del Cor; SIDIAP: Information system for the development of research in primary care

\section{Acknowledgment}

We thank the Catalan Health Institute and especially the SIDIAP Unit, which provided the database for the study. The authors also appreciate the English language review by Elaine Lilly, PhD, and are grateful to Carmen Ibáñez for administrative work.

\section{Funding}

This study was supported by a grant from the Ministry of Science and Innovation through the Instituto Carlos III (ISCiii) in the 2012 call for Strategic Health Action proposals under the National Plan for Scientific Research, Development and Technological Innovation 2008-2011; by the European Union through the European Regional Development Fund (IP12/00427); as part of the Primary Care Prevention and Health Promotion Research Network (rediAPP), by ISCiii-RETICS (RD12/0005). The funders had no role in the study design, collection, analysis and interpretation of data, writing of the manuscript or decision to submit for publication.

\section{Availability of data and materials}

The datasets are not available because researchers have signed an agreement with the Information System for the Development of Research in Primary Care (SIDIAP) concerning confidentiality and security of the dataset that forbids providing data to third parties. This organization is subject to periodic audits to ensure the validity and quality of the data.

\section{Authors' Contributions}

All authors contributed to the design of the study, revised the article, and approved the final version. CV and QFB drafted the article and obtained the funding. CV, NBR, QFB, ARL, EPR contributed to the analysis and interpretation of data. QFB and NBR wrote the first draft, and all authors (CV, NBR, QFB, ARL, EPR, MPV and MMM) contributed ideas, interpreted the findings and reviewed rough drafts of the manuscript. All authors read and approved the final manuscript.

\section{Competing interests}

The authors declare that they have no competing interests.

\section{Consent to publication}

Not applicable.

\section{Ethics approval and consent to participate}

The study protocol was approved by the Committee on the Ethics of Clinical Research, Institut Universitari d'Investigació en Atenció Primària Jordi Gol (IDIAP Jordi Gol) (Protocol No: P12/28). All data were anonymized and EHR confidentiality was respected at all times in accordance with national and international law.

\section{Author details}

'Institut Universitari d'Investigació en Atenció Primària Jordi Gol (IDIAP Jordi Gol), Gran Via Corts Catalanes, 587 àtic, 08007 Barcelona, Spain. ²Department of Paediatrics, Obstetrics and Gynaecology and Preventative Medicine. Facultad de Medicina, Universitat Autònoma de Barcelona, Edifici M. Campus Universitari UAB, 08193 Bellaterra (Cerdanyola del Vallès), Spain. ${ }^{3}$ Universitat Autònoma de Barcelona, Bellaterra (Cerdanyola del Vallès), Barcelona, Spain. ${ }^{4}$ Emergency Department, University Hospital of Vic, Francesc Pla el Vigatá, 08500 Vic Barcelona, Spain. ${ }^{5}$ Department of Medical Sciences, School of Medicine, University of Girona, Emili Grahit, 77, 17071 Girona, Spain. ${ }^{6}$ Faculty of Nursing, University of Girona, Emili Grahit, 77, 17071 Girona, Spain.

Received: 9 April 2016 Accepted: 18 October 2016

Published online: 03 November 2016

\section{References}

1. Violan C, Foguet-Boreu Q, Flores-Mateo G, Salisbury C, Blom J, Freitag M, et al. Prevalence, determinants and patterns of multimorbidity in primary care: a systematic review of observational studies. PLoS One. 2014;9(7): e102149.

2. United Nations, Department of Economic and Social Affairs, Population Division. World Population Ageing 2013. ST/ESA/SER.A/348. USA: United Nations publication; 2013.

3. Gijsen R, Hoeymans N, Schellevis FG, Ruwaard D, Satariano WA, van den Bos GA. Causes and consequences of comorbidity: a review. J Clin Epidemiol. 2001;54(7):661-74.

4. Wolff JL, Starfield B, Anderson GF. Prevalence, expenditures, and complications of multiple chronic conditions in the elderly. Arch Intern Med. 2002;162:2269-76.

5. Smith SM, Soubhi H, Fortin M, Hudon C, O'Dowd T. Managing patients with multimorbidity: systematic review of interventions in primary care and community settings. BMJ. 2012;345:e5205.

6. Foguet-Boreu Q, Violan C, Roso-Llorach A, Rodriguez-Blanco T, Pons-Vigués M, Muñoz-Pérez MA, et al. Impact of multimorbidity: acute morbidity, area of residency and use of health services across the life span in a region of south Europe. BMC Fam Pract. 2014;15:55.

7. WHO. Global status report on noncommunicable diseases 2014. Geneva: World Health Organization; 2015.

8. Mathur R, Pollara E, Hull S, Schofield P, Ashworth M, Robson J. Cardiovascular multimorbidity: the effect of ethnicity on prevalence and risk factor management. Br J Gen Pract. 2011;61(586):e262-70.

9. Prados-Torres A, Poblador-Plou B, Calderón-Larrañaga A, Gimeno-Feliu LA, González-Rubio F, Poncel-Falcó A, et al. Multimorbidity Patterns in Primary Care: Interactions among Chronic Diseases Using Factor Analysis. PLoS One. 2012;7(2):e32190.

10. Glynn LG, Buckley B, Reddan D, Newell J, Hinde J, Dinneen SF, Murphy AW. Multimorbidity and risk among patients with established cardiovascular disease: a cohort study. Br J Gen Pract. 2008;58(552):488-94.

11. Information system for the development of research in primary care (SIDIAP data base). [http://www.sidiap.org].

12. Del Mar García-Gil M, Hermosilla E, Prieto-Alhambra D, Fina F, Rosell M, Ramos $\mathrm{R}$, et al. Construction and validation of a scoring system for the selection of high-quality data in a Spanish population primary care database (SIDIAP). Qual Prim Care. 2012;20(2):135-45.

13. O'Halloran J, Miller GC, Britt H. Defining chronic conditions for primary care with ICPC-2. Fam Pract. 2004;21:381-6.

14. Defining Chronic Conditions for Primary Care Using ICPC-2. Available in: http://www.fmrc.org.au/Download/DefiningChronicConditions.pdf

15. Marrugat J, D'Agostino R, Sullivan L, Elosua R, Wilson P, Ordovas J, et al. An adaptation of the Framingham coronary heart disease risk function to European Mediterranean areas. J Epidemiol Community Health. 2003;57(8): 634-8.

16. Expert Panel on Detection, Evaluation, and Treatment of High Blood Cholesterol in Adults. Executive summary of the third report of the National Cholesterol Education Program (NCEP) Expert Panel on Detection, Evaluation, and Treatment of High Blood Cholesterol in Adults (Adult Treatment Panel III). JAMA. 2001;285:2486-97.

17. European Guidelines on cardiovascular disease prevention in clinical practice (version 2012). The Fifth Joint Task Force of the European Society of Cardiology and Other Societies on Cardiovascular Disease Prevention in 
Clinical Practice (constituted by representatives of nine societies and by invited experts). Eu Heart J. 2012:33(13):1635-701.

18. Egocheaga MI, Álvarez F, Alcázar R, Orte L, Gonzalez Parra E, Górriz JL, et al. Documento de consenso sobre la enfermedad renal crónica de la Sociedad Española de Nefrología (SEN) y de la Sociedad Española de Medicina de Familia y Comunitaria (semFYC). Barcelona: SEMFYC; 2007.

19. Violán C, Foguet-Boreu Q, Hermosilla-Pérez E, Valderas JM, FàbregasEscurriola M, Brugulat-Guiteras $\mathrm{P}$, et al. Comparison of the information provided by electronic health records data and a population health survey to estimate prevalence of selected health conditions and multimorbidity. BMC Public Health. 2013;13:251.

20. Fortin M, Hudon C, Haggerty J, Akker MV, Almirall J. Prevalence estimates of multimorbidity: a comparative study of two sources. BMC Health Serv Res. 2010;10:111

21. Raina P, Torrance-Rynard V, Wong M, Woodward C. Agreement between self-reported and routinely collected health-care utilization data among seniors. Health Serv Res. 2002;37:751-74.

22. Stewart M, Fortin M, Britt HC, Harrison CM, Maddocks HL. Comparisons of multi-morbidity in family practice-issues and biases. Fam Pract. 2013;30(4): $473-80$

23. Lowe D, Taylor M, Hill S. Changing definitions altered multimorbidity prevalence, but not burden associations, in a musculoskeletal population. J Clin Epidemiol. 2016;50895-4356(16):30029-4

24. Landwehr J, van den Akker M, Mersemakers JFM, Buntinx F. Comorbidity of chronic cardiovascular disorders. A cross-sectional analysis in a large general practice population in the Netherlands. Arch Public Health. 2000;58:213-31.

25. Oldridge NB, Stump TE, Nothwehr FK, Clark DO. Prevalence and outcomes of comorbid metabolic and cardiovascular conditions in middle- and olderage adults. J Clin Epidemiol. 2001;54(9):928-34.

26. Violán C, Foguet-Boreu Q, Roso-Llorach A, Rodriguez-Blanco T, Pons-Vigués M, Pujol-Ribera E, et al. Burden of multimorbidity, socioeconomic status and use of health services across stages of life in urban areas: a cross-sectional study. BMC Public Health. 2014;14:530

27. Prados-Torres A, Calderón-Larrañaga A, Hancco-Saavedra J, Poblador-Plou B, van den Akker M. Multimorbidity patterns: a systematic review. J Clin Epidemiol. 2014;67(3):254-66

28. Orueta JF, García-Álvarez A, García-Goñi M, Paolucci F, Nuño-Solinís R. Prevalence and Costs of Multimorbidity by Deprivation Levels in the Basque Country: A Population Based Study Using Health Administrative Databases. Schooling CM, ed. PLoS ONE. 2014;9(2):e89787.

29. Garin N, Olaya B, Perales J, Moneta MV, Miret M, Ayuso-Mateos JL, et al. Multimorbidity Patterns in a National Representative Sample of the Spanish Adult Population. PLoS One. 2014;9(1):e84794.

30. Nolasco A, Moncho J, Quesada JA, Melchor I, Pereyra-Zamora P, TamayoFonseca $\mathrm{N}$, et al. Trends in socioeconomic inequalities in preventable mortality in urban areas of 33 Spanish cities, 1996-2007 (MEDEA project). Int J Equity Health. 2015;14:33.

31. St Sauver JL, Warner DO, Yawn BP, Jacobson DJ, Mcgree ME, Pankratz JJ, et al. Why patients visit their doctors: assessing the most prevalent conditions in a defined American population. Mayo Clin Proc. 2013;88(1): 56-67.

32. Sicras-Mainar A, Velasco-Velasco S, González-Rojas Guix N, Clemente Igeño C, Rodríguez Cid JL. Influence of Morbidity, Metabolic Control, and Use of Resources in Subjects With Cardiovascular Risk in the Primary Care Setting. Aten Primaria. 2008:40:447-54.

33. Lobos Bejarano JR, Brotons CC. Cardiovascular risk factors and Primary Care: evaluation and intervention. Aten Primaria. 2011;43(12):668-77.

34. Barrios V, Escobar C, Echarri R. Concomitance of cardiovascular comorbidities in the hypertensive population: Not only in the United States. Arch Intern Med. 2008;168(12):1350-1.

35. Boyd CM, Leff B, Wolff JL, Yu Q, Zhou J, Rand C, et al. Informing clinical practice guideline development and implementation: Prevalence of coexisting conditions among adults with coronary heart disease. J Am Geriatr Soc. 2011;59(5):797-805

36. Carmona M, Garcia-Olmos LM, Alberquilla A, Munoz A, Garcia-Sagredo P, Somolinos $\mathrm{R}$, et al. Heart failure in the family practice: a study of the prevalence and co-morbidity. Fam Pract. 2011;28(2):128-33.
37. Stange KC. In this issue: Challenges of managing multimorbididy. Ann Fam Med. 2012;10:2-3.

38. Tisminetzky M, Goldberg R, Gurwitz JH. Magnitude and Impact of Multimorbidity on Clinical Outcomes in Older Adults with Cardiovascular Disease: A Literature Review. Clin Geriatr Med. 2016;32(2):227-46.

39. Salive ME. Future Research Directions for Multimorbidity Involving Cardiovascular Diseases. Clin Geriatr Med. 2016;32(2):399-407.

\section{Submit your next manuscript to BioMed Central and we will help you at every step:}

- We accept pre-submission inquiries

- Our selector tool helps you to find the most relevant journal

- We provide round the clock customer support

- Convenient online submission

- Thorough peer review

- Inclusion in PubMed and all major indexing services

- Maximum visibility for your research

Submit your manuscript at www.biomedcentral.com/submit
Biomed Central 